\title{
NOTA
}

\section{El principio de subsidiariedad en el proyecto europeo de la Comisión Delors'}

Carmen Pérez Linde ${ }^{2}$ y José María Margenat Peralta S.I. ${ }^{3}$

Resumen: La presidencia de la Comisión Europea por Jacques Delors entre 1985 y 1995 marcó un impulso profundo y duradero en el proceso de construcción del proyecto comunitario europeo. El principio de subsidiariedad jugó un papel clave para la Comisión Delors. Esta nota presenta el concepto de principio de subsidiariedad introducido por Jacques Delors. Como hipótesis de partida, se propone la siguiente: el reconocimiento y la aceptación del principio de subsidiariedad por los países de la Comunidad Económica Europea a partir de los años noventa permitió relanzar el proyecto europeo, que estaba bloqueado desde hacía más de una década. La nota describe el proceso seguido para la inclusión del principio de subsidiariedad en el Acta Única Europea y se analiza su extensión a otras políticas comunitarias a partir de las de medio ambiente; por último se describe el proceso de inclusión del principio de subsidiariedad en el Tratado de la Unión Europea desde un punto de vista político y jurídico.

Palabras clave: Acta Única Europea, Jacques Delors, principio de subsidiariedad, relanzamiento europeo, Tratado de la Unión Europea.

\footnotetext{
' Los autores agradecen los comentarios realizados por dos evaluadores anónimos.

${ }^{2}$ Licenciada en Ciencias Económicas y Empresariales por ETEA (2007), centro adscrito a la Universidad de Córdoba, y DEA por el mismo centro (2010). La nota recoge una parte del trabajo presentado para la obtención de la suficiencia investigadora, realizado bajo la dirección del profesor José $M$. Margenat.

${ }^{3}$ Profesor titular de Filosofía Social. Facultad de Ciencias Económicas y Empresariales - ETEA, Universidad de Córdoba.
} 
Fecha de recepción: 17 de diciembre de 2010.

Fecha de aceptación definitiva: 24 de marzo de 2011 .

\section{The principle of subsidiarity intro- duced in the EU Delors Commission project}

\begin{abstract}
Jacques Delors' presidency in the European Commission between 1985 and 1995 marked a profound and lasting impetus in the drafting of the European community project. The principle of subsidiarity played a key role in the Delors Commission. This paper presents the initial concept of subsidiarity introduced by Jacques Delors. As a hypothesis, we propose the following: that it was the recognition and acceptance of the principle of subsidiarity in European Economic Community countries in the nineties that opened the door to the relaunching of the European project, which had been blocked for more than a decade. The text describes the process behind the inclusion of the principle of subsidiarity in the European Single Act and discusses how it extended to other EU policies beginning with those pertaining to the environment. Finally it describes the inclusion of the subsidiarity principle in the European Union Treaty from a political and legal standpoint.
\end{abstract}

Key words: European relaunching, European Union Treaty, Jacques Delors, principle of subsidiarity, Single European Act.

\section{Le principe de subsidiarité dans le projet européen de la Commission Delors}

Résumé: La présidence de la Commission Européenne de Jacques Delors entre 1985 et 1995 fut un élan profond et durable dans le processus de construction du projet communautaire européen. Le principe de subsidiarité joua un rôle clé pour la Commission Delors. Ce texte présente le concept du principe de subsidiarité introduit par Jacques Delors. II propose, comme point de départ, l'hypothèse suivante la reconnaissance et l'acceptation du principe de subsidiarité par les pays de la Communauté Économique Européenne à partir des années quatre-vingt-dix a permis de relancer le projet européen, qui était bloqué depuis plus d'une décennie. Le texte décrit le processus suivi pourl'inclusion du principe de subsidiarité dans l'Acte Unique Européen, et analyse le processus d'extension à d'autres politiques communautaires à partir de celles del'environnement. Finalement, le texte décrit le processus d'inclusion du principe de subsidiarité dans le Traité de l'Union Européenne du point de vue politique et juridique.

Mots clés: Acte Unique Européenne, Jacques Delors, principe de subsidiarité, relance européenne, Traité de I'Union Européenne. 


\section{Introducción}

A pesar de que existen nociones del principio de subsidiariedad [en adelante, PS] en obras de pensadores como Aristóteles y Tomás de Aquino entre otros, la mayoría de los autores consideran que las raíces del PS moderno se hallan en la doctrina social católica de mediados del siglo XX. En 1931, en el contexto europeo de auge de los fascismos y otros estatalismos totalitarios, apareció una formulación explícita del PS en la encíclica Quadragesimo anno, donde Pío XI lo definía como una forma de organización que respeta siempre las responsabilidades más básicas de cada actor social. Una cita más amplia aclara esta definición:

Como no se puede quitar a los individuos y dar a la comunidad lo que ellos pueden realizar con su propio esfuerzo e industria, así tampoco es justo, constituyendo un grave perjuicio y perturbación del recto orden, quitar a las comunidades menores e inferiores lo que ellas pueden hacer y proporcionary dárselo a una sociedad mayor y más elevada, ya que toda acción de la sociedad, por su propia fuerza y naturaleza, debe prestar ayuda a los miembros del cuerpo social, pero no destruirlos y absorberlos. ${ }^{4}$

En 1951 se inició el proceso que llevó a la construcción de un proyecto comunitario europeo basado en una aplicación concreta, aunque aún implícita, del PS para establecer la paz entre los pueblos europeos. Los Estados-nación, como poderes absolutos o instancias superiores, ya no podían asegurar la paz y el bienestar de Europa actuando de forma independiente por ellos mismos, como atestiguaban los resultados de dos guerras mundiales.

Tras el discurso fundacional de RobertSchuman del 9 de mayo de 1950, se promovió el desarrollo de una instancia superior a los Estados-nación, capaz de establecer las bases para la paz y el bienestar tan ansiados en Europa. Esa instancia fue la Comunidad Económica del Carbón y del Acero (CECA), una comunidad subsidiaria respecto a los Estados firmantes del tratado de París (1951) por el que se establecía aquélla.

El proyecto europeo continuó progresando y asegurando estabilidad a los países que se iban integrando en él. A medida que nuevos países europeos mostraban su deseo de participar en la perceptiblemente ventajosa aventura de la construcción europea, se produjeron sucesivas ampliaciones de las Comunidades Europeas, más tarde de la Comunidad Europea y por último de la Unión Europea hasta llegar al momento presente.

${ }_{4}^{4}$ PíO XI (1931) Quadragesimo anno, http://www.vatican.va [Consulta: 1 de diciembre de 2010]. 
Durante todo este tiempo han existido épocas de desarrollo más o menos entusiasta del proyecto europeo y épocas de estancamiento y desánimo en la construcción del mismo, como sucedió al comienzo de los años setenta, cuando la crisis económica internacional provocada por la repentina y drástica elevación de los precios del petróleo, frenó el progreso económico de las Comunidades Europeas y bloqueó el desarrollo de la idea que los padres fundadores de Europa habían puesto en marcha. Un periodo de desánimo y mantenimiento rutinario de lo conseguido siguió hasta mediados los años ochenta.

Jacques Delors, que fue presidente de la Comisión Europea de 1985 a 1995, "redescubrió" el PS, explicitándolo y aplicándolo, modestamente al principio y cada vez en más ámbitos hasta llegar a generalizar su uso como herramienta fundamental en los procesos de toma de decisiones (Tratado de la Unión Europea, 1993).

Desde entonces el PS ha resultado ser una auténtica palanca en manos de los distintos órganos de gobierno comunitario y de los países miembros (a veces con puntos de vista diferentes e incluso encontrados) que, en general, ha servido y sirve bien a los objetivos de una cada vez más cohesionada Unión Europea.

El objeto de esta nota es presentar el uso del PS, que Jacques Delors hizo durante su mandato, en favor del proyecto comunitario europeo. Proponemos, como hipótesis de partida, esta interpretación: el reconocimiento y la aceptación del PS por los países miembros de la Comunidad Económica Europea a mediados de los años ochenta permitió relanzar el proyecto europeo, estancado desde hacía más de una década.

Esta nota está ordenada en cuatro apartados, tras esta introducción. El segundo apartado describe el proceso seguido para la inclusión de la filosofía del PS en el Acta Única Europea si bien en aquel momento sólo en el área de medio ambiente. El tercero se dedica al proceso por el que el PS se extendió a diversas políticas comunitarias y al debate provocado sobre los poderes de la Comunidad Económica Europea. El cuarto describe el proceso de inclusión del PS en el Tratado de la Unión Europea como principio político y como principio jurídico. En el quinto se recogen las conclusiones del trabajo. Al final se relaciona la bibliografía utilizada.

\section{El proceso hacia el Acta Única Europea}

El panorama económico europeo de los primeros años ochenta no era alentador. En Estados Unidos se acuñaron los términos europesimismo o euroesclerosis para 
describir la decadencia económica europea de los años setenta y primera mitad de los años ochenta. ${ }^{5}$

El mercado común que debía haberse completado en 1970 seguía inacabado en 1985. La Comunidad Económica Europea (CEE) había trabajado durante años para eliminar las barreras arancelarias, olvidando otras no arancelarias que también obstaculizaban la libertad de mercado entre los países miembros. Éstas no sólo se mantuvieron durante este período sino que incluso aumentaron debido al crecimiento del proteccionismo nacional, estimulado por la crisis económica: barreras técnicas, numerosas reglamentaciones nacionales que diferían significativamente de un país a otro, prácticas anticompetitivas, que las autoridades públicas toleraban o promovían, y otras actuaciones contrarias al libre mercado. ${ }^{6}$ Todas ellas dificultaban o impedían la libre circulación de productos y servicios.

A mediados de los años ochenta, cuando la crisis económica comenzó a superarse, pareció haber llegado un tiempo propicio para impulsar nuevamente el proyecto comunitario europeo. El político francés Jacques Delors, comenzó su mandato como presidente de la Comisión Europea el 7 de enero de 1985. Comprometido con el objetivo de relanzar la construcción europea, Delors había estudiado los posibles medios para ello y pensó en la idea de promover la defensa común europea, pero la descartó pronto como medio de relanzamiento porque vio que ese tema no estaba aún maduro y podía provocar bloqueos por parte de los países miembros. Pensó en acometer una reforma institucional, pero fue consciente de que ciertos países miembros se mostrarían reticentes a ella; todos eran conscientes de que nadie estaba dispuesto a un procedimiento de toma de decisiones que no fuera el de unanimidad. Pensó también en un refuerzo del Sistema Monetario Europeo, pero por lo menos dos países pondrían la condición previa de la libertad de movimiento para los capitales en el espacio europeo.

Delors llegó a la conclusión de que el relanzamiento del proyecto europeo tenía que consistir precisamente en conseguir el objetivo del Tratado de Roma de 1957: el logro de un mercado común, esta vez como un verdadero mercado interior. Había que superar todos los obstáculos que impidieron su realización tiempo atrás y suprimir por completo la noción de frontera entre los países miembros

${ }^{5}$ Cfr. J. DeloRs (1992) Le nouveau concert Européen, París, Odile Jacob, 22.

${ }^{6}$ Cfr. D. Fernández Navarrete (2007) Fundamentos económicos de la Unión Europea, Madrid, Thomson, 64-75. 
para asegurar la libertad de circulación de mercancías. El Consejo aprobó esta propuesta y encargó a la Comisión un programa y un calendario preciso para conseguir el Objetivo 92, es decir, completar el mercado interior o mercado único para finales de 1992.

Pocos meses después de hacer público ese encargo, el 14 de junio de 1985, la Comisión presidida por Delors respondió al Consejo con la publicación del Libro blanco sobre el mercado interior, que fue aprobado por el Consejo Europeo reunido en Milán los días 28 y 29 de junio de 1985.7

Delors, la presidencia italiana de aquel Consejo y varios ministros eran conscientes de que sin una modificación del Tratado de Roma no se podría alcanzar el mercado único. Por este motivo fue convocada en dicho Consejo de Milán una conferencia intergubernamental (en adelante, CIG) en la que se negociarían las modificaciones de los tratados constitutivos de París y de Roma. La CIG fue desarrollándose a lo largo del verano y del otoño de 1985. Los informes resultantes fueron presentados en el Consejo Europeo de Luxemburgo el 2 y el 3 de diciembre de 1985, donde más tarde, el 17 de febrero de 1986, se procedió a firmar un nuevo tratado, el Acta Única Europea (en adelante, AUE), que fue la primera revisión de los tratados constitutivos. Entró en vigor el día 1 de julio de 1987.

El AUE resultó ser un tratado corto y de interpretación clara. Su texto mantuvo la unicidad del esquema institucional. Con ello se mostraba que tanto la cooperación en política exterior (que se incorporaba por primera vez en un tratado y que sólo consistió en una modesta formalización de la cooperación política ya existente entre los países miembros), como la revisión de los tratados constitutivos, debían ser consideradas como un único instrumento para el relanzamiento y progreso del proyecto comunitario.

Entre otras medidas, se estableció para muchos asuntos la toma de decisiones en el Consejo de ministros por mayoría cualificada -lo que permitió agilizar su funcionamiento-, aumentó algo la participación del Parlamento en la toma de decisiones ${ }^{8}$ se fijaron medidas orientadas a la consecución de una Europa sin fronteras en un plazo que había de finalizar el 31 de diciembre de 1992, se reforzó el Sistema Monetario Europeo, se fortaleció el marco comunitario en cooperación

${ }^{7}$ Cfr. J. Delors (2004) Mémoires, París, Plon, 207.

${ }^{8}$ Cfr. A. MoravcsiK (1998) The choice for Europe, social purpose and state power from Messina to Maastricht, Ithaca (Nueva York), Cornell University, 320-321. 
tecnológica, se establecieron las bases para las políticas de cohesión económica y social y, por último, se instauró una política comunitaria de medio ambiente inspirada en la filosofía del PS.

Esta política medioambiental fue motivo de discordia, pues había países miembros que la deseaban, aunque otros, como Dinamarca, ya habían dado sus primeros pasos en ese ámbito nacional. Gracias a la aceptación del PS, se atenuaron los temores y se suavizaron las disconformidades de los Estados y de los entes territoriales que los conformaban, pues se aprobó que, en principio, la protección del medio ambiente era competencia de los países miembros. La Comunidad intervendría de manera subsidiaria en el momento en que esa protección pudiera realizarse mejor a nivel comunitario.

Aquella crisis económica internacional de los años setenta y ochenta hizo que cada país se concienciara de sus límites. Los países europeos comprendieron que, por si mismos, no eran ya capaces de asegurar el bienestar económico de sus propios pueblos, dado lo limitado de sus mercados nacionales. La solución más sensata era acogerse a la propuesta de Delors, la creación de un verdadero mercado único comunitario, basado naturalmente en el PS, que estimulara las empresas comunitarias, recompensara el espíritu de innovación, ofreciera la dimensión indispensable para permitir que las economías se fortalecieran?..., y que, en definitiva, trajera la prosperidad económica a los países miembros.

\section{El proceso hacia el Tratado de la Unión Europea}

La adopción del Objetivo 92 y la aprobación del AUE, que sentó los principios de organización del mercado único, fueron los primeros pasos para el relanzamiento de la construcción europea. La inclusión de la filosofía del PS para el área de medio ambiente en el nuevo tratado tuvo pocas consecuencias políticas. Pero con el transcurso del tiempo y el desarrollo de los medios necesarios para llevar a cabo las disposiciones del AUE, el principio se fue extendiendo a otras áreas distintas. Esto fue generando un debate sobre los poderes que iba adquiriendo la Comunidad. No fue de extrañar que Gran Bretaña, concretamente su entonces primera ministra Margaret Thatcher que había llevado a cabo un exhaustivo programa de reducción del intervencionismo estatal, saliera a la palestra intentando provocar el interés de la opinión pública por los nuevos y rápidos cambios de Europa.

${ }^{9}$ Cfr. J. Delors (1992) Le nouveau concert Européen, París, Odile Jacob, 9. 
El mejor exponente de ese debate fue el discurso de 1988 de Thatcher en el Colegio de Europa de Brujas, en el que acusó al conjunto de instituciones europeas de intervencionismo excesivo. Un año más tarde Delors contestó al discurso de Thatcher en el mismo lugar. A continuación exponemos los puntos más significativos que enfatizan aquel contraste de opiniones:

\section{CUADRO I. Debate sobre los poderes de la Comunidad europea: aportaciones de Margaret Thatcher y Jacques Delors}

\begin{tabular}{|c|c|}
\hline Margaret Thatcher - 20-IX-1988 & Jacques Delors - 17-X-1989 \\
\hline $\begin{array}{l}\text { La Comunidad Europea es la cooperación } \\
\text { voluntaria y activa entre Estados soberanos } \\
\text { e independientes. }\end{array}$ & $\begin{array}{l}\text { La Comunidad Europea es una comunidad de } \\
\text { naciones que ejercen una soberanía conjunta, } \\
\text { que hablan con una sola voz. }\end{array}$ \\
\hline $\begin{array}{l}\text { La Comunidad ha de reflejar las tradiciones, } \\
\text { las aspiraciones y el orgullo nacional de cada } \\
\text { país miembro. }\end{array}$ & $\begin{array}{l}\text { El modelo federalista europeo no va contra } \\
\text { las tradiciones, las aspiraciones o el orgullo } \\
\text { nacional de los distintos países miembros. } \\
\text { El principio federalista de subsidiariedad } \\
\text { permite la emergencia de una Europa unida } \\
\text { y la fidelidad a la nación. }\end{array}$ \\
\hline $\begin{array}{l}\text { En asuntos monetarios, el tema clave no es si } \\
\text { debiera haber un Banco Central Europeo. }\end{array}$ & $\begin{array}{l}\text { Es primordial que exista un Banco Central } \\
\text { Europeo que defina una política monetaria } \\
\text { común. Su estructura federal garantiza que } \\
\text { cada banco central nacional participe en la } \\
\text { toma de decisiones colectivas y luego apli- } \\
\text { que a su nivel, con sustanciales márgenes } \\
\text { de maniobra, esas decisiones adoptadas } \\
\text { conjuntamente. }\end{array}$ \\
\hline $\begin{array}{l}\text { Las necesidades inmediatas y prácticas en } \\
\text { asuntos monetarios son: establecer un com- } \\
\text { promiso de la Comunidad con el movimiento } \\
\text { libre de capitales y establecer un mercado } \\
\text { libre de servicios financieros. }\end{array}$ & $\begin{array}{l}\text { Un movimiento libre de capitales precisa } \\
\text { de una política monetaria común. De lo } \\
\text { contrario, la Comunidad se enfrentaría a la } \\
\text { especulación internacional y a la inestabili- } \\
\text { dad de las monedas dominantes. }\end{array}$ \\
\hline $\begin{array}{l}\text { Se ha de facilitar el paso de viajeros y de } \\
\text { bienes a través de toda la Comunidad, } \\
\text { pero sin abolir totalmente los controles de } \\
\text { las fronteras internas, con el fin de proteger } \\
\text { a los ciudadanos comunitarios del crimen y } \\
\text { detener el movimiento de drogas, terroristas } \\
\text { e inmigrantes ilegales. }\end{array}$ & $\begin{array}{l}\text { La Comisión ejecutará la voluntad del Consejo } \\
\text { Europeo de eliminar las fronteras internas } \\
\text {-físicas, técnicas y fiscales-y de ofrecer a los } \\
\text { ciudadanos europeos una gran área para el } \\
\text { comercio, encuentros y asociaciones. }\end{array}$ \\
\hline
\end{tabular}




\begin{tabular}{|c|c|}
\hline Margaret Thatcher - 20-IX-1988 & Jacques Delors - 17-X-1989 \\
\hline \multirow{3}{*}{$\begin{array}{l}\text { Las regulaciones en el mercado de trabajo } \\
\text { han de ser mínimas. Han de ampliarse } \\
\text { los mercados y reducir la intervención del } \\
\text { gobierno. } \\
\text { No necesitamos nuevas regulaciones que } \\
\text { eleven el costo del empleo y que hagan que } \\
\text { el mercado de mano de obra europeo sea } \\
\text { menos flexible y menos competitivo con los } \\
\text { suministradores de fuera. }\end{array}$} & $\begin{array}{l}\text { El mercado de trabajo tiene necesidad de } \\
\text { regulaciones: }\end{array}$ \\
\hline & $\begin{array}{l}\text { - Es necesaria la Carta de los Derechos } \\
\text { Sociales, cuyo objetivo es repetir que } \\
\text { la Comunidad no intenta subordinar } \\
\text { los derechos fundamentales de los } \\
\text { trabajadores únicamente a la eficiencia } \\
\text { económica. }\end{array}$ \\
\hline & $\begin{array}{l}\text { Nuestras compañías necesitan un marco } \\
\text { legal, opcional por supuesto, que facilite } \\
\text { la cooperación y la armonización y que } \\
\text { las capacite para hacer frente a los retos } \\
\text { del gran mercado y de la competición } \\
\text { nacional. }\end{array}$ \\
\hline
\end{tabular}

Fuentes: M. ThatCher (1988) "Discurso en el Colegio de Europa de Brujas, 20 de septiembre", <http:// www.ena.lu/address given margaret thatcher_bruges 20 september_1988-2-9492> [Consulta: 4 de enero de 2010] y J. Delors (1989) "Discurso en el Colegio de Europa en Brujas del 17 de octubre", $<$ http://www.ena.lu/address given jacques delors bruges 17 october 1989-2-10171> [Consulta: 30 de noviembre de 2009].

Elaboración: C. PÉREZ LINDE

En este debate sobre los poderes de la Comunidad también participaron los "Länder" de Alemania, aunque no con tanta intensidad como Gran Bretaña. Aquéllos temían una intromisión de la Comunidad en sus competencias regionales exclusivas. Delors se dirigió a ellos especialmente en su discurso pronunciado en Bonn el 5 de octubre de 1989 y les expuso que la Comunidad seguía un modelo federalista que no aspiraba a centralizar el poder, y que gracias al principio federal de subsidiariedad era posible la emergencia de una Europa unida y la fidelidad a la propia nación y sus entes territoriales.

Se había llegado a un momento en el que la Comunidad tenía importantes responsabilidades internacionales. Ciertos países no pertenecientes a la Comunidad esperaban mucho de ella, sobre todo los del centro y del este de Europa. La aceleración de la historia provocada por la caída de la Unión Soviética, la unificación alemana y los planes de creación de una Unión Económica y Monetaria exigían a la Comunidad una urgente actuación de naturaleza política. 
La necesidad de cambios en el tratado se hacía evidente. En un Consejo Europeo celebrado en Dublín en abril de 1990 se previó la convocatoria de dos CIG en diciembre de ese mismo año, una sobre la Unión Económica y Monetaria y otra sobre una Unión Política cuyo fin fuese fortalecer la legitimidad democrática de la Comunidad.

\section{El Tratado de la Unión Europea}

El 15 de diciembre de 1990 se iniciaron en Roma las dos CIG mencionadas. Las negociaciones de ambas se desarrollaron paralelamente con el fin de redactar un tratado cuya aprobación por el Consejo Europeo diera lugar a una nueva entidad, la Unión Europea. El 7 de febrero de 1992, en Maastricht, aquellas dos CIG concluyeron con la firma del Tratado de la Unión Europea. El día 1 de noviembre de 1993 entró en vigor el TUE tras ser ratificado por cada uno de los Estado-miembro.

El Tratado de la Unión Europea o Tratado de Maastricht modificó el Tratado de París de 1951 por el que se creó la Comunidad Económica del Carbón y del Acero (CECA), los dos Tratados de Roma de 1957 por los que se crearon la Comunidad Económica Europea (CEE) y la Comunidad Europea de la Energía Atómica (EURATOM) y el Acta Única Europea de 1986 (AUE) por el que las tres comunidades anteriores pasaron a formar la Comunidad Europea. Con el Tratado de la Unión Europea (en adelante, TUE), la Comunidad Europea pasó a ser la Unión Europea.

El TUE se estructura en tres pilares: un pilar comunitario y dos de cooperación intergubernamental. La principal diferencia entre ellos es que, en los pilares de cooperación intergubernamental las políticas no emanan de las instituciones europeas, sino que son sistemas de cooperación de las políticas de los Estadosmiembro, y por tanto en ellos se da una escasa participación de las instituciones europeas. Esta estructura de tres pilares o de templo griego, fue muy discutida durante las CIG previas al TUE. La Comisión Delors defendió que el proyecto de tratado debía mantener la unicidad de políticas, como el AUE, pero se quedó sin apoyos en las negociaciones y el proyecto de tratado adoptó la estructura del "templo griego". 10

$10 \mathrm{~J}$. Delors (1994) L'unité d'un homme (Entretiens avec Dominique Wolton), París, Odile Jacob, 271-273. 
El primer pilar del TUE está formado por los tratados constitutivos y sus reformas, las instituciones europeas, sus logros al canzados -principalmente, el mercado único, la Unión Económica y Monetaria, la Política Agraria Común, los fondos estructurales y los fondos de cohesión- y las novedades que el TUE introduce en este primer pilar: el reconocimiento del principio de subsidiariedad, el reconocimiento de una ciudadanía europea, la concreción de las fases de la Unión Económica y Monetaria, el refuerzo de la cohesión económica y social mediante fondos estructurales, un procedimiento de codecisión del Parlamento por el que éste en ciertas materias pueda decidir junto al Consejo Europeo, un refuerzo de las competencias del Tribunal de Justicia y del Tribunal de Cuentas, la creación del Comité de las Regiones, con carácter consultivo, y el aumento de competencias en la política económica y monetaria, la educación y la formación profesional, la cultura, la salud pública, la protección de consumidores, las redes transeuropeas, la industria, la cohesión económica y social, la investigación y el desarrollo tecnológico, el medio ambiente y la cooperación al desarrollo. El segundo pilar es el ámbito de la política exterior y de seguridad común (PESC). El tercer pilar es el de la cooperación en asuntos de justicia y los asuntos de interior (CAJl). ${ }^{11}$

En las negociaciones de las dos CIG previas a la firma del TUE, los representantes de los Estados-miembro insistieron en la necesidad de explicitar el PS en el nuevo tratado. Interpretado como un principio de mínima interferencia, el PS limitaría la expansión de poderes comunitarios provocada por la generalización del voto por mayoría cualificada en el Consejo (aprobado en el AUE). Los gobiernos nacionales no consideraban conveniente prescindir de ese voto, fundamentalmente por razones de eficacia y de supervivencia del proyecto europeo, pero querían ponerle un contrapeso. El resultado de aquellas insistencias fue la consagración del PS en el TUE como un principio político y como un principio jurídico.

El preámbulo y el Artículo A del TUE hacen referencia a la naturaleza política del PS: se trata más bien de una regla de sentido común para el reparto constitucional de competencias, las enunciadas en el tratado, o las necesarias para conseguir un objetivo previsto en el tratado, son comunitarias y han de ser ejercidas por la Comunidad. No obstante, es posible que algunas competencias comunitarias puedan ser ejercidas por los Estados-miembro siempre que, respetando los principios generales y las disposiciones comunitarias, puedan hacerlo de modo suficiente. La falta de cumplimiento de esta regla no está sujeta a un control jurídico.

1 D. López GarRIDo (1992) El Tratado de Maastricht, Madrid, Closas-Orcoyen, 25-31. 
El artículo 3B del Tratado de la Comunidad Europea (que se incluye en el TUE) hacía referencia a un PS de naturaleza jurídica. El PS sirve para discernir si una competencia no exclusiva de la Comunidad debe ser ejercida por la Comunidad o por el Estado. La competencia no comunitaria debe ejercerla en principio el Estado, siempre que éste sea suficiente para alcanzar los objetivos y se preserve la proximidad al ciudadano. En caso de que se pruebe la insuficiencia del Estado, y la Comunidad pueda alcanzar mejor los objetivos, intervendrá la Comunidad. La decisión que se tome está sujeta a un control jurídico posterior del Tribunal de Justicia de la Comunidad.

La introducción del PS en el TUE produjo una ampliación de competencias comunitarias, tales como las políticas social, regional, de competencia, de investigación y desarrollo y de medio ambiente. También dio lugar a un traspaso de competencias de los Estados-miembro a la Unión Europea. Dicho traspaso fue más cualitativo que cuantitativo, lo que quiere decir que la Unión Europea reguló, cada vez de una forma más común, aspectos concretos de cada política como la creación de redes transeuropeas, la política de visados, las políticas culturales, la salud pública, la protección de los consumidores, la política educativa, la política de cooperación para el desarrollo, la política industrial, el reconocimiento de una verdadera ciudadanía, la política exterior y de seguridad común o, por último, la política interior y de justicia, antes que establecer una política común en cada materia para los Estados-miembro, como fue en el caso de la política monetaria. ${ }^{12}$

Esta fue una etapa muy importante para el proyecto de construcción europea, pues no sólo se terminó de configurar la integración económica sino que la Comunidad inició un proceso de integración política. Después de la entrada en vigor del TUE en noviembre de 1993 y del fin del mandato de Delors como presidente de la Comisión Europea en diciembre de 1994, tuvo lugar la cuarta ampliación de la Comunidad a 15 miembros en enero de 1995: Austria, Finlandia y Suecia se adhirieron al proyecto comunitario. En el TUE se habían dejado preparadas las bases para futuros cambios, pues contaba con una cláusula de revisión por la que se preveía la convocatoria en 1996 de una CIG para su propia reforma. Aquella conferencia llegó a celebrarse, resultando de ella el Tratado de Ámsterdam de 1997.

La Unión Europea, ha seguido creciendo y avanzando. Hasta ahora sus veintisiete miembros han luchado como una unidad frente a la crisis económica internacional,

${ }^{12}$ E. MURILIO GARCía (1999) "Al gunas reflexiones sobre el reparto de competencias entre las instituciones comunitarias y los Estados de la Unión: ¿Más responsabilidad para Europa?": Revista de Fomento Social 54 (1999) 524-525. 
apoyando y rescatando "economías enfermas" de países miembros. En el mes de mayo de 2010 se creó el Fondo Europeo de Estabilidad Financiera para rescatar la economía de Grecia. Este fondo se ha vuelto a emplear en el pasado mes de noviembre de 2010 para rescatar la economía de Irlanda, y cabe la posibilidad de que este "dispositivo de ayuda de emergencia" vuelva a emplearse para otros rescates financieros ${ }^{13}$. En la lucha por la supervivencia económica en un contexto global, el PS sigue aplicándose con claridad y mostrando su eficacia.

\section{Conclusión}

A mediados de los años ochenta se iniciaron en Europa una serie de cambios, tras el estancamiento precedente y el funcionamiento rutinario de la CEE. Eran cambios para mejorar la construcción europea, aumentar el nivel de integración de los países miembros, ampliar el número de éstos, aceptando a todos los que cumplieran unos mínimos requisitos políticos y económicos, crear una unión política y económica que hiciera de Europa una potencia mundial de primera línea que fuera capaz de generar desarrollo y bienestar para sus habitantes, competir económicamente con las restantes potencias mundiales establecidas y "emergentes", colaborar políticamente en el mantenimiento de la paz entre los pueblos del mundo y contribuir de manera efectiva al desarrollo y bienestar general.

Europa no esperaba conseguir a corto o a medio plazo tan ambiciosos objetivos, que ni siquiera estaban en la opinión ciudadana o en la mayoría de los políticos y pensadores europeos. Pero algunos europeístas no habían olvidado las aspiraciones de los padres fundadores. La tarea se presentaba delicada y difícil, y se requería visión, estrategia, táctica y decisión para llevarla a cabo.

Éstas son las conclusiones a que nos ceñimos, después del anterior recorrido. Jacques Delors, político europeísta francés que ejerció la presidencia de la Comisión Europea durante casi diez años, fue el artífice del relanzamiento europeo en la década de los ochenta. Su herramienta clave fue el principio de subsidiariedad. Él pensó que la inclusión de este principio en los tratados constitutivos brindaría una nueva oportunidad de crecimiento a la Comunidad Europea. Y efectivamente así fue. La inclusión del principio en el Acta Única Europea y en el Tratado de la

13 Cfr. B. CASSEN (2010) "El Consenso de Berlín se impone en Europa": Le Monde diplomatique (en español) n ${ }^{\circ} 182,3$. En el momento de redactarse estas líneas, aún no se había producido el "rescate" de Portugal ni la segunda intervención griega.

Revista de Fomento Social 66 (201I) 
Unión Europea proporcionó una oportunidad de crecimiento no sólo para beneficio de la Comunidad, sino también para todos sus Estados-miembro.

El modelo europeo es un modelo federalista muy peculiar, donde el nivel supranacional complementa los niveles nacional, regional y local. Los niveles no se sustituyen. Además, todas las instancias de poder están unidas gracias al principio de subsidiariedad, que asigna y reasigna las competencias a los distintos niveles.

El principio de subsidiariedad es un poderoso instrumento de organización para el funcionamiento cotidiano de sociedades complejas. Su correcta utilización mejora la eficacia y la eficiencia de la labor de gobierno. Permite un acercamiento de la toma de decisiones al ciudadano, ahorra costes y permite que grupos sociales más o menos complejos reciban una atención administrativa adecuada y suficiente aunque no tengan capacidad para proporcionársela por ellos mismos.

\section{Bibliografía}

BreÑA, R. (2000) "El principio de Subsidiariedad y la Construcción de una Europa Social": Foro Internacional, 161, pp. 467-483.

CASSEN, B. (2010) "El Consenso de Berlín se impone en Europa": Le Monde diplomatique (en español), núm. 182, p. 3.

Chicharro Lázaro, A. (2001) El Principio de subsidiariedad en la Unión Europea, Zizur Menor (Navarra), Aranzadi, 324 págs.

COMISIÓN EUROPEA (1989) "Informe sobre la Unión Económica y Monetaria en la Comunidad Europea, Luxemburgo", http://aei.pitt.edu/1007/01/monetary delors.pdf [Consulta: 3 de marzo de 2010]

Consejo Europeo (1992) "Consejo Europeo de Edimburgo, 11 y 12 de diciembre de 1992, Conclusiones de la Presidencia", <http://www.cepc.es/rap/Publicaciones/ Revistas/5/RIE 020001 369.pdfs [Consulta: 14 de abril de 2010]

Consejo Europeo (1990a) "Consejo Europeo de Roma, 14 y 15 de diciembre de 1990, Conclusiones de la Presidencia", http://www.cepc.es/rap/Publicaciones/ Revistas/5/RIE 018001 383.pdf [Consulta: 10 de marzo de 2010] 
Consejo Europeo (1990b) "Consejo Europeo de Roma, 27 y 28 de octubre de 1990, Conclusiones de la Presidencia", http://www.cepc.es/rap/Publicaciones/ Revistas/5/RIE 017003 281.pdf [Consulta: 3 de marzo de 2010]

Consejo Europeo (1990c) "Consejo Europeo de Dublín, 25 y 26 de junio de 1990, Conclusiones de la Presidencia", http://www.cepc.es/rap/Publicaciones/ Revistas/5/RIE 017002 365.pdf [Consulta: 13 de febrero de 2010]

Consejo Europeo (1990d) "Consejo Europeo de Dublín, 28 de abril de 1990, Conclusiones de la Presidencia", http://www.cepc.es/rap/Publicaciones/Revistas/5/ RIE 017001 295.pdf [Consulta: 24 de enero de 2010]

Consejo Europeo (1989a) "Consejo Europeo de Estrasburgo, 8 y 9 de diciembre de 1989, Conclusiones de la Presidencia", hittp://www.cepc.es/rap/Publicaciones/ Revistas/5/RIE 016003 388.pdf [Consulta: 20 de enero de 2010]

Consejo Europeo (1989b) "Consejo Europeo de Madrid, 26 y 27 de junio de 1989, Conclusiones de la Presidencia", http://www.cepc.es/rap/Publicaciones/ Revistas/5/RIE_016_002_283.pdf [Consulta: 15 de enero de 2010]

Consejo Europeo (1988) "Consejo Europeo de Hannover, 27 y 28 de junio de 1988, Conclusiones de la presidencia", http://www.cepc.es/rap/Publicaciones/ Revistas/5/RIE 015002 333.pdf [Consulta: 22 de diciembre de 2009]

Consejo Europeo (1985) "Consejo Europeo de Bruselas, 29 y 30 de marzo de 1985, Conclusiones de la Presidencia", hitp://www.cepc.es/rap/Publicaciones/ Revistas/5/RIE 012001 359.pdf [Consulta: 20 de diciembre de 2009]

Delors, J. y Dolú, M. (2009) Investir dans le social, París, Odile Jacob, 284 pp.

DeloRs, J. (2006) L'Europe tragique et magnifique, les grands enjeux européens, París, Saint-Simon, $189 \mathrm{pp}$.

DeloRs, J. (2004) Mémoires, París, Plon, 511 pp.

DeloRs, J. (1994) L'unité d'un homme (Entretiens avec Dominique Wolton), París, Odile Jacob, 397 pp.

DeloRs, J. (1992) Le nouveau concert Européen, París, Odile Jacob, 349 pp. 
Delors, J. (1989) "Discurso en el Colegio de Europa en Brujas del 17 de octubre", http://www.ena.lu/address given jacques delors bruges 17 october 1989-210171 [Consulta: 30 de noviembre de 2009].

ElaZAR, D. (1991) "The European Community: between state sovereignty and subsidiarity", http://www.jcpa.org/dje/articles2/eurcomm.htm [Consulta: 25 de noviembre de 2009]

Fernández Navarrete, D. (2007) Fundamentos económicos de la Unión Europea, Madrid, Thomson, $411 \mathrm{pp}$.

FONSECA, F.J. y MARTIN, J.A. (1992) "La Unión Europea: génesis de Maastricht": Revista de Instituciones Europeas, núm. 2, vol. 19, pp. 517-564.

López GarRIDO, D. (1992) El Tratado de Maastricht, Madrid, Closas-Orcoyen, 433 pp.

MORAVCSIK, A. (1998) The choice for Europe, social purpose and state power from Messina to Maastricht, Ithaca (Nueva York), Cornell University, 514 pp.

MurILL GarcíA, E. (1999) "Algunas reflexiones sobre el reparto de competencias entre las instituciones comunitarias y los Estados de la Unión: ¿̇Más responsabilidad para Europa?": Revista de Fomento Social 54, pp. 519-535.

Pío XI (1931) Quadragesimo Anno.

PontiFICIO COnSEJo JusticIA Y PAZ (2005) Compendio de la Doctrina Social de la Iglesia, Madrid y Barcelona, Biblioteca de Autores Cristianos - Planeta, 420 pp.

RodríGuez-IzQuierdo, M. (2008) Posibilidades constitucionales del principio de subsidiariedad. Granada, Universidad de Granada, 174 pp.

ThatCher, M. (1988) "Discurso en el Colegio de Europa en Brujas del 20 de septiembre", http://www.ena.lu/address given margaret thatcher bruges 20 september 1988-2-9492 [Consulta: 4 de enero de 2010] 